\title{
Preservation of Beam Emittance in the Presence of Ion Motion in Future High-Energy Plasma-Wakefield-Based Colliders
}

\author{
R. Gholizadeh, ${ }^{1}$ T. Katsouleas, ${ }^{2}$ P. Muggli, ${ }^{1}$ C. Huang, ${ }^{3}$ and W. Mori ${ }^{3}$ \\ ${ }^{1}$ University of Southern California, Los Angeles, California 90089, USA \\ ${ }^{2}$ Duke University, Durham, North Carolina 27708, USA \\ ${ }^{3}$ University of California, Los Angeles, California 90095, USA \\ (Received 14 December 2009; published 16 April 2010)
}

\begin{abstract}
The preservation of beam quality in a plasma wakefield accelerator driven by ultrahigh intensity and ultralow emittance beams, characteristic of future particle colliders, is a challenge. The electric field of these beams leads to plasma ions motion, resulting in a nonlinear focusing force and emittance growth of the beam. We propose to use an adiabatic matching section consisting of a short plasma section with a decreasing ion mass to allow for the beam to remain matched to the focusing force. We use analytical models and numerical simulations to show that the emittance growth can be significantly reduced.
\end{abstract}

Ultralow emittance beams are required for future high energy colliders. These beams allow for the strong focusing at the interaction point to produce extremely small transverse beam sizes, thereby increasing the collision rate between counterpropagating beam particles and maximizing the collider luminosity and the probability of discovery. Electron beams with low emittances can be produced by radio-frequency (rf) or photoinjector guns [1]. Ultralow emittances are reached in damping rings where the emittance is lowered by synchrotron radiation [2]. Cooling of hadron beams requires other techniques such as electron [3], stochastic [4], or coherent cooling [5]. The challenge for any accelerator is to preserve the emittance of the incoming beam. In conventional rf accelerators, the main source of emittance growth is the small and random misalignment of magnetic optical elements.

In a beam driven plasma-based accelerator, or plasma wakefield accelerator (PWFA), a short electron bunch (drive bunch) generates a wake by displacing the plasma electrons off its path. The plasma electrons are then pulled back by the background plasma ions, and create a large longitudinal electric field that can accelerate a second bunch (witness bunch), if injected at the right time. Previous work has shown that the emittance of the incoming witness bunch is well preserved when the drive bunch density is of the order of the plasma density [6]. In this case, the accelerated (witness) bunch propagates in a pure column of immobile ions with a focusing force that increases linearly with radius. The plasma acts as an ideal focusing element, free of geometric aberrations, and preserves the incoming beam emittance. In addition, emittance growth due to scattering on the plasma ions has been shown to be negligible for light ions plasmas [7]. However, the high bunch charge (nanocoulombs) and ultralow emittances of the witness bunch required for future accelerators lead to bunches with densities much larger than the plasma density. The high density arises from the need to match the bunch to the plasma focusing force (i.e., small transverse size) in order to avoid large oscillations of the beam envelope and excessive synchrotron radiation loss [8]. The bunch must also be short (a few tens of microns) to be accelerated with narrow energy spread [6]. The drive bunch is discarded at the end of the acceleration process, and its emittance and matched transverse size can thus be larger than those of the witness bunch. However, it also needs to be short and carry a high charge to excite large wakefields. As a result of the corresponding very large bunch space charge fields, the plasma ions move during a single plasma wave period, even though they are much heavier that the mobile plasma electrons. This ion motion destroys the uniform ion column linear focusing field and results in a nonlinear focusing force that can lead to a significant emittance growth [9-11].

Several techniques to reduce the emittance growth in a PWFA have been proposed [12]. Gholizadeh et al. [13] introduced the concept of nonlinear transverse beam profile preservation, which, in theory, can eliminate the emittance growth. However, in practice, designing bunches with arbitrary density profiles is challenging. The amount of plasma ions motion is inversely proportional to the ions mass [9-11], and is therefore reduced in plasmas with heavy ions. However, nuclear scattering is then the new source of emittance growth in long plasmas [11].

In this Letter, we introduce the concept of adiabatic matching of the witness beam to the nonlinear focusing field of the ion channel. We show that it can alleviate the emittance growth due to plasma ions motion caused by the drive beam. In this concept, the beam is injected in a plasma section with an initially large ion mass. The ion mass is then adiabatically reduced along the plasma that leads to the accelerating section. We determine the optimum ion mass profile to minimize the emittance growth. We then show simulation results for a beam with parameters typical of the future International Linear Collider 
(ILC) to illustrate how the matching section can significantly reduce the emittance growth.

In the adiabatic approach, the incoming electron bunch with Gaussian transverse profile enters a plasma matching section with a heavy ion mass so that the ions do not move significantly over a plasma period. Since the bunch density is much larger than the plasma density, the accelerated bunch propagates in a pure ion column with linear focusing force [11]. The incoming Gaussian beam profile is matched to the initial focusing force. The mass of the ions is then slowly reduced along the plasma, hence allowing the plasma ions to move and the focusing force to only slowly become nonlinear and thus the transverse bunch profile to slowly evolve and remain matched to the nonlinear focusing force. Towards the end of the matching section the ion mass reaches that of the accelerating section (e.g., lithium). The adiabatic change in the focusing force reduces the emittance growth compared to that in plasma with a low ion mass. In the next section, we find an analytic solution for the optimal mass profile for the adiabatic matching section.

In the plasma wakefield accelerator (PWFA), the accelerating wake is driven by a short ultrarelativistic particle bunch. For a given (geometric) emittance $\varepsilon$, the transverse size or beta function $\beta=\sigma^{2} / \varepsilon$ of an electron beam matched to the linear focusing force $F$ of a uniform ion column is given by $\beta^{2}=\gamma m c^{2} /(F / r)$ [14]. Using Gauss' law to determine the column electric field, the focusing force of the uniform single-ionized ion column with density $n_{0}$ is $F(r)=-2 \pi n_{0} e^{2} r[9,10]$.

For a round bunch, the plasma ions motion is symmetrical about the beam axis, and one can use an ion ring model [9] to determine the column density. For a ring of ions of initial radius $r_{0}$ and collapsing to a radius $r<r_{0}$ when reaching the tail of the bunch (one $\sigma_{z}$ after the bunch peak density, $\sigma_{z}$ the rms length of the Gaussian bunch profile), the ring density is

$$
n=\frac{n_{0} r_{0}^{2}}{r^{2}} .
$$

Using the harmonic oscillator model for the ions that move due to the electric field of the drive electron bunch $[10,11], r$ is given by

$$
r=r_{0} \cos \left(\frac{k_{\mathrm{H}} \sqrt{2 \pi} \sigma_{z}}{\sqrt{M}}\right)
$$

in which $k_{\mathrm{H}}=\sqrt{\frac{r_{a} N_{b} \sqrt{r_{e} n_{0} \gamma}}{A_{\mathrm{H}} \varepsilon_{n, x} \sigma_{z}}}$ is the oscillation constant in a hydrogen ion plasma, $r_{a}=1.55 \times 10^{-18} \mathrm{~m}$ is the classical radius of a singly charged ion of mass $1 \mathrm{amu}, r_{e}=2.8 \times$ $10^{-15} \mathrm{~m}$ is the classical radius of electron, $N_{b}$ is the number of particles in the witness bunch, $n_{0}$ is the background plasma density, $A_{\mathrm{H}}$ is the mass of hydrogen atom ( $1 \mathrm{amu}), \varepsilon_{n, x}$ and $\sigma_{z}$ are the normalized emittance and longitudinal size of the witness bunch, respectively, and
$M=A / A_{\mathrm{H}}$ is the atomic mass of the plasma ions. For the adiabatic matching section we make the ion mass a function of the longitudinal coordinate $s$ along the plasma $M=$ $M(s)$. First we assume that $M(s)$ changes continuously. Therefore the density of the ions at the tail of the drive bunch in the presence of ion motion is [from Eqs. (1) and (2)]

$$
n=\frac{n_{0}}{\cos ^{2}\left(\frac{k_{\mathrm{H}} \sqrt{2 \pi} \sigma_{z}}{\sqrt{M(s)}}\right)} .
$$

Once the ions pass the tail of the drive bunch, they are no longer pulled in but rather repelled by the space charge of the other ions. In order to simplify the problem of finding the focusing force on the witness bunch, we assume that the maximum ion collapse happens at the tail of the drive bunch (in reality, the ion collapse continues for a short distance after the tail due to the transverse momentum). Therefore, the ion density at the witness bunch is assumed to be the same as the ion density at the tail of the drive bunch. Using this assumption and Eq. (3), the beta function of the bunch matched to the collapsed ion column density is

$$
\beta=\left(\frac{\gamma m c^{2}}{2 \pi n_{0} e^{2}}\right)^{1 / 2} \cos \left(\frac{k_{\mathrm{H}} \sqrt{2 \pi} \sigma_{z}}{\sqrt{M(s)}}\right) .
$$

The assumption of adiabaticity [13] requires that $|d \beta / d s| \ll 1$. Applying this condition to Eq. (4) results in

$$
\left|-\left(\frac{\gamma m c^{2}}{2 \pi n_{0} e^{2}}\right)^{1 / 2} \frac{k_{\mathrm{H}} \sqrt{2 \pi} \sigma_{z}}{M^{3 / 2}(s)} \sin \left(\frac{k_{\mathrm{H}} \sqrt{2 \pi} \sigma_{z}}{\sqrt{M(s)}}\right) \frac{d M(s)}{d s}\right| \ll 1 .
$$

Note that in the above equations, the energy of bunch is assumed to remain constant. We will later verify this assumption by showing that the matching section is short compared to the accelerating section. Starting with a large ion mass and with $M(s)$ a decreasing function of $s$ $(d M / d s<0)$, we obtain

$$
-\frac{d M(s)}{d s} \ll\left(\frac{2 \pi n_{0} e^{2}}{\gamma m c^{2}}\right)^{1 / 2} \frac{M^{3 / 2}(s)}{k_{\mathrm{H}} \sqrt{2 \pi} \sigma_{z} \sin \left(\frac{k_{\mathrm{H}} \sqrt{2 \pi} \sigma_{z}}{\sqrt{M(s)}}\right)} .
$$

For example, taking the left-hand side as $10 \%$ of the right-hand side of Eq. (6) and inserting in the parameters for a drive bunch with typical ILC parameters $\left(N_{b}=1.5 \times\right.$ $10^{10}, \sigma_{z}=35 \mathrm{um}, \varepsilon_{n}=4 \times 10^{-6} \mathrm{~m}-\mathrm{rad}, \gamma=10^{6}$ ) [10] and a plasma density $n_{e}=0.9 \times 10^{16} \mathrm{~cm}^{-3}$ appropriate to reach accelerating gradients in excess of $10 \mathrm{GeV} / \mathrm{m}$ [15] leads to

$$
\frac{d M(s)}{d s}=-0.013 \frac{M^{3 / 2}(s)}{\sin \left(\frac{6.45}{\sqrt{M(s)}}\right)}
$$




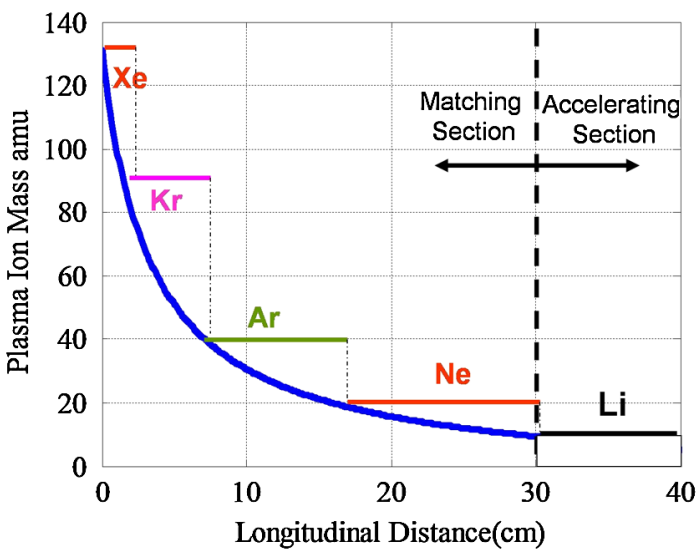

FIG. 1 (color online). Optimal ion mass profile for the matching section, continuous and in discrete steps using noble gases.

whose solution is plotted in Fig. 1 for an initial mass $M_{0}=$ 131 (xenon) and a mass $M_{f}=7$ (lithium) for the accelerating section. The matching section in this case is only about $30 \mathrm{~cm}$ long. In practice, however, one cannot decrease the ions mass continuously, but rather in several shorter sections with discrete commonly available gases. Figure 1 also depicts a possible discontinuous profile using five atomic elements. The energy gain in the $30 \mathrm{~cm}$-long matching section is only about $15 \mathrm{GeV}$ (at $50 \mathrm{GeV} / \mathrm{m}$ average accelerating gradient), which is small compared to the beam energy ( $500 \mathrm{GeV}-1 \mathrm{TeV})$, verifying the a priori assumption that energy gain can be neglected in this section.

In order to simulate the results, we use the simplified model described in Ref. [12]. In this model, the bunch is assumed to be traveling inside a pure ion column. The electric fields of the ions and of the bunch are obtained from Gauss' law and the witness bunch evolves in the calculated focusing force.

Figure 2 shows the focusing force on the witness bunch at the end of the four discrete steps of the matching section, and after $10 \mathrm{~cm}$ of the accelerating section (Fig. 1). Since

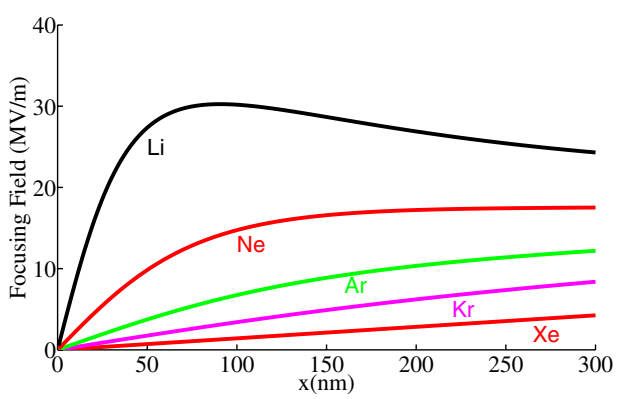

FIG. 2 (color online). Focusing fields at the end of the steps of the discrete matching section of Fig. 1. Note that the witness bunch's matched transverse size and initial emittance are $\sigma_{x}=$ $140 \mathrm{~nm}$ and $\varepsilon_{x}=1 \times 10^{-7} \mathrm{~m}-\mathrm{rad}$, respectively. the heavy ions are almost immobile in the initial plasma, the focusing force remains linear. As the ions become lighter, the focusing force slowly becomes nonlinear towards the end of the matching section. Initially, the bunch has a Gaussian density profile [Fig. 3(a)] matched to the initial linear focusing force (Fig. 2) and remains matched as it slowly evolves along the plasma as a result of the slow change in ion mass. Note that the bunch profile at the end of the accelerating section [no matching section Fig. 3(b)] is very similar to that obtained in simulations and experiments with positron bunches in plasmas [16], as the two cases involve the same physics.

The evolution of witness bunch is calculated in three cases. In case 1, the bunch travels directly through the accelerating section with a light ion lithium plasma. In cases 2 and 3, the bunch travels through the matching section with a continuous and a discrete ion mass change of Fig. 1, respectively, before entering the accelerating section. Figure 3(a) shows the initial phase space of the initial bi-Gaussian bunch. Figure 3(b) shows the bunch phase space after $40 \mathrm{~cm}$ of lithium plasma. The focusing force becomes immediately nonlinear and the phase space area, and therefore also the bunch emittance increase significantly, by a factor of 11 (Fig. 4). Figure 3(c) shows the phase space and the bunch profile after the ideal, continuous matching section. In this case, the bunch smoothly evolves and particles are no longer spread over a large phase space area, i.e., the emittance growth is much smaller, only about $20 \%$ (Fig. 4). Figure 3(d) shows the phase space at the end of the more realistic discrete matching section. As expected, the phase space area (i.e., the emittance) is slightly greater than the optimal continuous matching section, a factor of 2 (Fig. 4). Note that in cases 2 and 3 , the bunch is focused by the matching section and the profile remains close to Gaussian [Figs. 3(c) and 3(d)]. This demonstrates a very significant emittance growth
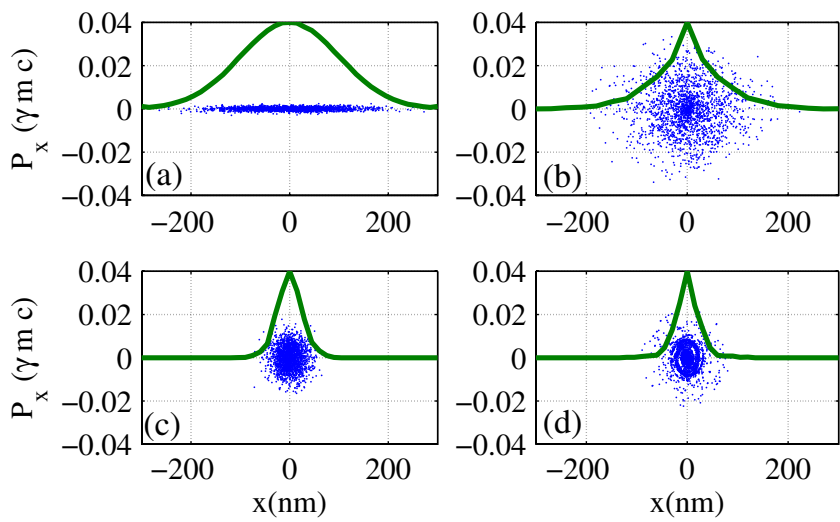

FIG. 3 (color online). Phase space of the beam: (a) initial biGaussian, (b) $30 \mathrm{~cm}$ into the accelerating section (no matching section), (c) after a $30 \mathrm{~cm}$ matching section and $10 \mathrm{~cm}$ of accelerating section, and (d) after the $30 \mathrm{~cm}$ discrete matching section and $10 \mathrm{~cm}$ of accelerating section. The corresponding transverse beam profiles are also shown. 


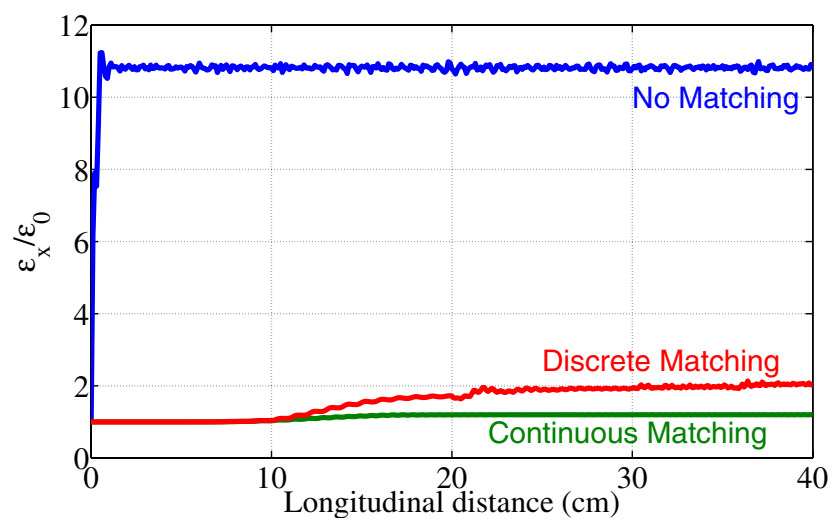

FIG. 4 (color online). Emittance growth for accelerating only case, the continuos matching section, and the discretely matching section case.

reduction provided by the adiabatic matching method and its potential impact on a future plasma-based collider.

It is worth mentioning that multiple ionization (not taken into account here) may be an issue when using higher mass plasma ions. The force between the ions and bunch electrons is directly proportional to the ions ionization state, and in practice, multiple ionization could counter the benefit of using heavier ions. However, our preliminary results show that multiple ionization happens mostly around the witness bunch (since the drive bunch can be made larger and therefore have a lower electric field), and that the newly created electrons do not leave the witness bunch volume immediately since they are in the accelerating region of the wakefield. Therefore, most of the new born electrons get trapped and dragged along [17], which compensates for the excess positive charge from the new born ions. Future work will include multiple ionization of the plasma ions and its effect on the beam emittance growth minimization and on the wakefields themselves.
In summary, we found an analytic formula for the optimal mass profile of an adiabatic matching section. We showed that an ideal adiabatic matching section with continuous mass change can greatly reduce the undesirable emittance growth created by ion motion in a future high energy plasma wakefield accelerator.

The authors would like to thank USC High Performance Computations \& Communications (HPCC). This work is supported by Department of Energy Contract No. DEFG02-92ER40745.

[1] R. Brinkmann et al., Phys. Rev. ST Accel. Beams 4, 053501 (2001).

[2] P. Emma et al., Phys. Rev. ST Accel. Beams 4, 021001 (2001).

[3] I. N. Meshkov, Phys. Part. Nucl. 25, 631 (1994).

[4] S. van der Meer, Rev. Mod. Phys. 57, 689 (1985).

[5] V. N. Litvinenko and Y. S. Derbenev, Phys. Rev. Lett. 102, 114801 (2009).

[6] P. Muggli et al., Phys. Rev. Lett. 93, 014802 (2004).

[7] N. Kirby et al., in Proceedings of the Particle Accelerator Conference, Albuquerque, New Mexico, 2007 (IEEE, Albuquerque, NM, 2007), p. 4183.

[8] G. Blumenthal et al., Rev. Mod. Phys. 42, 237 (1970); D. K. Johnson et al., Phys. Rev. Lett. 97, 175003 (2006).

[9] S. Lee et al., AIP Conf. Proc. 472, 524 (1999).

[10] J. Rosenzweig et al., Phys. Rev. Lett. 95, 195002 (2005).

[11] R. Gholizadeh et al., AIP Conf. Proc. 877, 504 (2006).

[12] R. Gholizadeh et al., in Proceedings of the Particle Accelerator Conference, Albuquerque, New Mexico, 2007 (IEEE, Albuquerque, NM, 2007), pp. 3067-3069.

[13] R. Gholizadeh et al., AIP Conf. Proc. 1086, 575 (2009).

[14] T. Katsouleas et al., AIP Conf. Proc. 230, 134 (1991).

[15] S. Lee et al., Phys. Rev. ST Accel. Beams 5, 011001 (2002).

[16] P. Muggli et al., Phys. Rev. Lett. 101, 055001 (2008).

[17] E. Oz et al., Phys. Rev. Lett. 98, 084801 (2007). 